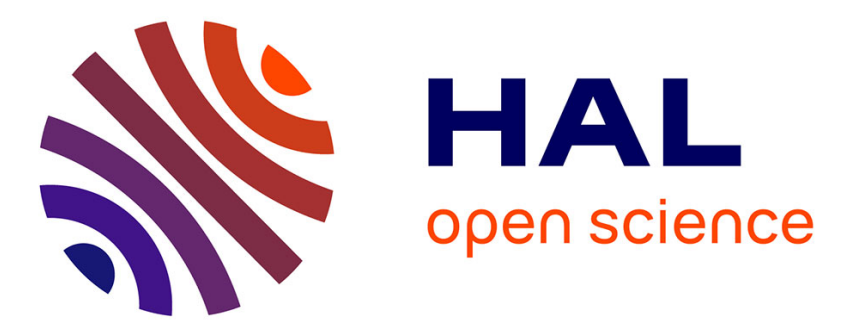

\title{
Path following control of unmanned quadrotor helicopter with obstacle avoidance capability
}

Zhixiang Liu, Laurent Ciarletta, Chi Yuan, Youmin Zhang, Didier Theilliol

\section{To cite this version:}

Zhixiang Liu, Laurent Ciarletta, Chi Yuan, Youmin Zhang, Didier Theilliol. Path following control of unmanned quadrotor helicopter with obstacle avoidance capability. International Conference on Unmanned Aircraft Systems, ICUAS'17, Jun 2017, Miami, Florida, United States. hal-01537732

\section{HAL Id: hal-01537732 \\ https://hal.science/hal-01537732}

Submitted on 6 Jul 2017

HAL is a multi-disciplinary open access archive for the deposit and dissemination of scientific research documents, whether they are published or not. The documents may come from teaching and research institutions in France or abroad, or from public or private research centers.
L'archive ouverte pluridisciplinaire HAL, est destinée au dépôt et à la diffusion de documents scientifiques de niveau recherche, publiés ou non, émanant des établissements d'enseignement et de recherche français ou étrangers, des laboratoires publics ou privés. 


\title{
Path Following Control of Unmanned Quadrotor Helicopter with Obstacle Avoidance Capability
}

\author{
Zhixiang Liu ${ }^{1}$, Laurent Ciarletta ${ }^{1}$, Chi Yuan ${ }^{2}$, Youmin Zhang ${ }^{2}$, and Didier Theilliol ${ }^{3}$
}

\begin{abstract}
This paper proposes a new path following methodology combining with a obstacle avoidance scheme for unmanned quadrotor helicopter (UQH) capable of working in the cluttered and hazardous environments. A new cross-track error prediction based mechanism, where the cross-track error is estimated by utilizing the extend Kalman filter (EKF), is first developed for the path following scheme. Then, the UQH is equipped with obstacle avoidance capability employing a lightcomputational approach, the visibility graph algorithm. The priority of UQH is to switch to obstacles avoidance maneuvering in the presence of obstacles, and continue to execute the assigned mission after avoiding all hazardous objects blocking the desired path. The control system developed for attitude and position control of UQH is also introduced. Finally, extensive simulation studies on a nonlinear model of UQH with a series of dangerous scenarios are conducted to demonstrate the effectiveness of the proposed methodology.
\end{abstract}

\section{INTRODUCTION}

The last decades have seen a tremendous progress in the development of unmanned aerial vehicles (UAVs). A growing number of research institutes, universities, governments, and commercial entities across the world are developing and employing UAVs for a diverse range of applications, such as scientific research [1], environmental monitoring and surveillance [2], [3], natural resources exploration [4], post-disaster search and rescue [5], and military missions [6]. Unmanned quadrotor helicopters (UQHs), as an important group of $\mathrm{UAV}$, possess tremendous advantages comparing with other manned/unmanned aerial vehicles including affordable cost of development [7], decreased mechanical structure complicacy [8], easy-to-fly [9], enhanced maneuverability and outstretched deployability [10], [11], as well as experimental platform for newly developed techniques [12].

As the complexities of application grow, UQHs are accordingly required to deploy in more cluttered, sophisticated, and hazardous environments, this situation strongly demands $\mathrm{UQHs}$ to be equipped with enhanced reliable and safe instruments as well as more robust guidance and control algorithms for guaranteeing satisfactory performance of tasks execution and preventing collision with buildings, mountains, trees, other manned/unmanned vehicles, etc.. However, semiautonomy up to date is still favored over full autonomy due to

\footnotetext{
${ }^{1}$ Zhixiang Liu and Laurent Ciarletta are with the Lorraine Research Laboratory in Computer Science and its Applications (LORIA), University of Lorraine, Nancy, France, 1zhx180@gmail.com, laurent.ciarletta@loria.fr

${ }^{2}$ Chi Yuan, and Youmin Zhang are with the Department of Mechanical and Industrial Engineering, Concordia University, Montreal, Canada, chi_yua@encs.concordia.ca, ymzhang@encs.concordia.ca

${ }^{3}$ Didier Theilliol with CRAN, University of Lorraine, Nancy, France, didier.theilliol@univ-lorraine.fr
}

the diverse nature of missions and limited obstacle avoidance capabilities of UQHs. Therefore, this work is intended to establish the study of methodology with satisfactory and safe performance to improve the autonomy of current UQHs including increasing application diversity and minimizing the amount of human supervision.

The existing mission execution can be generally partitioned into three predominant sorts: set-point stabilization, trajectory tracking, and path following [13]. This study falls into the path following problem of UQH. From the literature, there exist two categories of design methods: 1) individual design of guidance and control systems, which separates the system into an inner control loop and an outer guidance loop; 2) integrated design of guidance and control systems. In order to simplify the design procedure and separately manage the guidance-related disturbances and uncertainties problems in the guidance system without introducing them to control system and complicating the design and debugging procedures, this study chooses to separately design guidance and control systems. Numerous existing research works have been dedicated to the field with individual design philosophy. In [14], the robust nonlinear control theory is used for maneuvering a UQH to follow a class of smooth Jordan curves. [15] presents a modified purepursuit path following method which uses inertial velocity in the calculation of commanded lateral acceleration. With this method, both straight and curved line paths can be well followed. A nonlinear output-feedback control method, which employs the global exponential observer, Lyapunov's direct method and backstepping technique, is developed in [16] for path following application. In [17], a vector-field based path following scheme is devised to generate desired course references to attitude control in the inner-loop system. [18] introduces a path following control law which relies on a nonlinear control strategy derived at the kinematic level.

Regarding obstacle avoidance methods, a large number of heuristic obstacle avoidance methods have been developed for UAVs, such as particle swarm optimization (PSO) [19], genetic algorithm (GA) [20], artificial potential field (APF) [21], [22], and probabilistic roadmap-based method [23]. Considering the low computational capability of the onboard processor of UQH as well as high maneuverability of $\mathrm{UQH}$, this study selects a light-computational obstacle avoidance method, the visibility graph [24], as the obstacle avoidance design.

Following the concept of hierarchical structure design, a hybrid system, which consists of three functionality modules including the path following, obstacle avoidance, and motion 


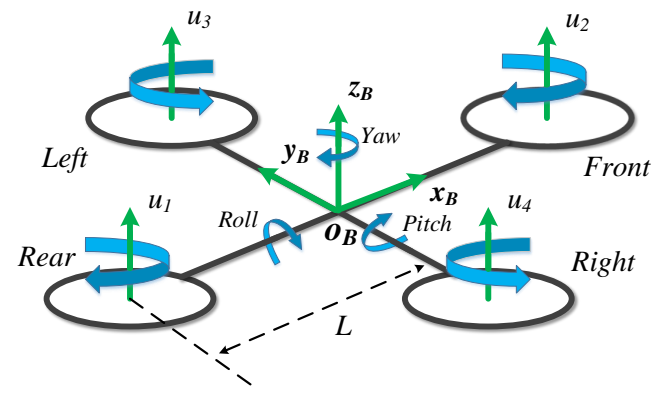

Fig. 1: Schematic diagram of the UQH.

control, is developed in this study. The first module of the proposed strategy is devised for operating the UQH to follow the desired path with satisfactory performance. To improve the performance of path following, a path following method with the capability of predicting cross-track error is developed; the cross-track error is estimated by an extended Kalman filter (EKF). The second module is designed for obstacle avoidance by modifying the reference heading angle to control system when there are any obstacles blocking the desired path. In order to significantly reduce the excessive computation burden of onboard processing devices, a relatively simple but effective method, the visibility graph, is utilized for the design of obstacle avoidance mechanism. The last module is the motion control system which maneuvers $\mathrm{UQH}$ to follow the reference path assigned from the first and second modules. The traditional proportional-integralderivative (PID) control approach is used for the height maintenance, while a fuzzy proportional-derivative (FPD) control method is chosen for operating the attitude of $\mathrm{UQH}$, expecting that the UQH is capable of adapting with different working conditions. Finally, numerical simulations on a nonlinear UQH model is carried out to verify the efficacy of the proposed methodology.

The remainder of this paper is arranged as follows: Section 2 provides some preliminaries for the design of proposed methods. Section 3 introduces the design details of proposed approaches. Section 4 addresses the conducted numerical simulations and their corresponding performance analyses. The last section summarizes the conclusions and potential future works.

\section{PReliminaries}

\section{A. Nonlinear Model of Unmanned Quadrotor Helicopter}

The UQH, as concisely depicted in Fig. 1, is normally actuated by four motor-driven propellers which are located at its four corners (front, rear, left, and right, respectively). These propellers generate corresponding thrusts $u_{1}, u_{2}, u_{3}$, and $u_{4}$. The motion of UQH can be briefly illustrated as follows: 1) distributing identical amount of control signal to each motor to obtain the vertical translation; 2) assigning a different amount of control signals to the opposite motors to achieve the horizontal translation [7].

As described in [7], a common UQH dynamical model with respect to the earth-fixed coordinate system can be
TABLE I: Nomenclature (earth-fixed coordinate system)

\begin{tabular}{ll}
\hline Symbols & Explanation \\
\hline$x, y, z$ & Coordinates of UQH at center of mass \\
$\theta$ & Pitch angle \\
$\phi$ & Roll angle \\
$\psi$ & Yaw angle \\
$u_{z}(t)$ & Total lift force \\
$u_{\theta}(t)$ & The applied torque in $\theta$ direction \\
$u_{\phi}(t)$ & The applied torque in $\phi$ direction \\
$u_{\psi}(t)$ & The applied torque in $\psi$ direction \\
$K_{n}(n=1,2, \ldots, 6)$ & Drag coefficients \\
$u_{i}(t)(i=1,2,3,4)$ & Thrust of each rotor \\
$L$ & Distance of UQH's gravity and propeller \\
$C_{m}$ & Thrust-to-moment scaling factor \\
$g$ & Acceleration of gravity \\
$m$ & UQH mass \\
$I_{x}$ & Moment of inertia along $x$ direction \\
$I_{y}$ & Moment of inertia along $y$ direction \\
$I_{z}$ & Moment of inertia along $z$ direction \\
$\omega_{m}$ & Actuator bandwidth \\
$K_{m}$ & A positive gain \\
$u_{c i}(t)(i=1,2,3,4)$ & PWM signals distributed to each rotor \\
\hline
\end{tabular}

written as follows:

$$
\begin{aligned}
\ddot{x} & =\frac{(\sin \psi \sin \phi+\cos \psi \sin \theta \cos \phi) u_{z}(t)-K_{1} \dot{x}}{m} \\
\ddot{y} & =\frac{(\sin \psi \sin \theta \cos \phi-\cos \psi \sin \phi) u_{z}(t)-K_{2} \dot{y}}{m} \\
\ddot{z} & =\frac{(\cos \theta \cos \phi) u_{z}(t)-K_{3} \dot{z}}{m}-g \\
\ddot{\phi} & =\frac{u_{\phi}(t)-K_{4} \dot{\phi}}{I_{x}} \\
\ddot{\theta} & =\frac{u_{\theta}(t)-K_{5} \dot{\theta}}{I_{y}} \\
\ddot{\psi} & =\frac{u_{\psi}(t)-K_{6} \dot{\psi}}{I_{z}} .
\end{aligned}
$$

Furthermore, from the relationship between accelerations and lift/torques, one can obtain that:

$$
\left[\begin{array}{c}
u_{z}(t) \\
u_{\theta}(t) \\
u_{\phi}(t) \\
u_{\psi}(t)
\end{array}\right]=\left[\begin{array}{cccc}
1 & 1 & 1 & 1 \\
L & -L & 0 & 0 \\
0 & 0 & L & -L \\
C_{m} & C_{m} & -C_{m} & -C_{m}
\end{array}\right]\left[\begin{array}{l}
u_{1}(t) \\
u_{2}(t) \\
u_{3}(t) \\
u_{4}(t)
\end{array}\right] .
$$

The propeller force and its corresponding pulse width modulation (PWM) signal have the following relationships:

$$
u_{i}(t)=K_{m} \frac{\omega_{m}}{s+\omega_{m}} u_{c i}(t) .
$$

The above-mentioned symbols are all defined in Table I.

\section{Path Following Scheme Design}

As outlined in Fig. 2, the path following and control schemes are separately designed, for the purpose of avoiding any errors defecting the reference heading angle before introducing it to the control system.

\section{A. Cross-Track Error Prediction Based Path Follow Rule}

In order to successfully follow the assigned path, UQV is required to move through a set of predefined waypoints along the path with a constant forward speed. As illustrated in 


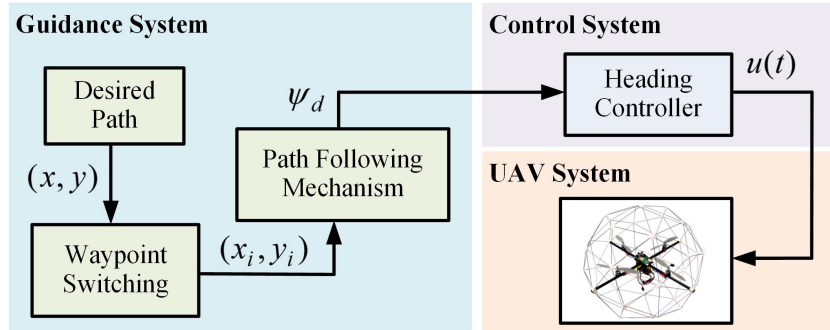

Fig. 2: Illustration of the proposed path following scheme.

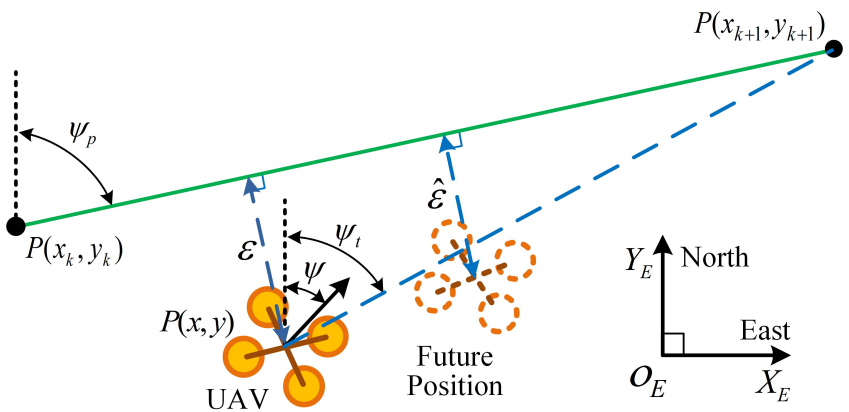

Fig. 3: Geometry of the proposed path following scheme.

Fig. 3, a two-dimensional (2D) continuous $\mathcal{C}^{1}$ parametrized path, which is chosen in this study, goes through a set of successive waypoints $P\left(x_{k}, y_{k}\right)$ for $k=1, \ldots, i$.

For an arbitrary position of UAV locates at $P(x, y)$, the cross-track error, which is defined as the orthogonal distance to the path tangential reference frame, is calculated by:

$$
\varepsilon=\frac{\left(y_{k+1}-y_{k}\right) x+\left(x_{k}-x_{k+1}\right) y+y_{k} x_{k+1}-x_{k} y_{k+1}}{\sqrt{\left(y_{k+1}-y_{k}\right)^{2}+\left(x_{k}-x_{k+1}\right)^{2}}},
$$

where $P\left(x_{k}, y_{k}\right)$ and $P\left(x_{k+1}, y_{k+1}\right)$ denote the last and next waypoints, respectively.

In order to follow the desired path, a reference heading angle is required. Before calculating this reference angle, in this study, a prediction of cross-track error is first defined as follows:

$$
\hat{\varepsilon}=\varepsilon+U_{f} \times T_{s} \times \sin (\Delta \psi),
$$

where $\hat{\varepsilon}$ is the prediction of cross-track error, $U_{f}$ represents the forward velocity of $\mathrm{UAV}, T_{s}$, which denotes the updating rate, is selected to calculate $\hat{\varepsilon}$ for the next period. $\Delta \psi=$ $\psi_{r e f}-\psi$ is the error between the path-tangential angle $\psi_{p}$ and real heading angle $\psi$ of UAV. The path-tangential angle $\psi_{p}$ is constant when the reference path is straight, while $\psi_{p}$ varies in each sampling time when the desired path is a curve.

Based on the prediction of cross-track error, the reference heading angle $\psi_{d}$ can then be obtained:

$$
\psi_{d}=\psi_{p}+K_{c} \hat{\varepsilon},
$$

where $K_{c}$ is a coefficient used for tuning the conversion from cross-track error to reference heading angle.

In practice, however, the fixed gains $T_{s}$ and $K_{c}$ can be well tuned for rapidly reducing the cross-track error, while an undesirable fluctuation around the reference path may be caused, vice versa. Thereby the prediction of cross-track error is intended to be redesigned for the purpose of fast decreasing the cross-track error, while avoiding the unexpected oscillation around the path as well. The improvement to Eq. (6) is then made by designing the following saturation function:

$$
f(\hat{\varepsilon})=\frac{T_{1} \hat{\varepsilon}}{|\hat{\varepsilon}|\left(1+e^{-T_{2}\left(|\hat{\varepsilon}|-T_{3}\right)}\right)},
$$

where $T_{1}, T_{2}$, and $T_{3}$ are selected according to the practical situation including forward speed.

It is worth-mentioning that the introduction of saturation function to the prediction of cross-track error can improve the performance of path following from two aspects: 1) guarantee a more rapid operation of UAV approaching to the desired path when it is far away from the path; 2) smoothly manoeuvre the UAV to follow the desired path when it is close to the path.

Consequently, Eq. (6) with combination of Eq. (7) becomes:

$$
\psi_{d}=\psi_{p}+K_{c} f(\hat{\varepsilon}) .
$$

\section{B. Improving Path Following Rule with EKF}

Although Eq. (5) provides a solution of predicting the cross-track error, an improvement is still desirable due to the fact that this kinematics based method tends to be sensitive to environmental disturbances, noises, and various of uncertainties. In this study, EKF is further employed to modify the design of prediction. The reason behind this selection is EKF's advantage in estimation based on its statistical characteristic, and it has also been popularly and successfully applied in a variety of practical applications [25].

First, the following system model and measurement model are established [26]:

$$
\left\{\begin{aligned}
x_{i} & =f\left(x_{i-1}\right)+\omega_{i-1}, \\
d_{i} & =h\left(x_{i}\right)+\nu_{i},
\end{aligned}\right.
$$

where the stochastic variable $x_{i}$ is the system state at time $t_{i} . \omega_{i-1}$ and $\nu_{i}$ denote the process and measurement noises, respectively. $f(\cdot)$ and $h(\cdot)$ represent the nonlinear vector functions of the states.

Then, the problem introduced by EKF is to find an estimate $\hat{x}_{i}$ of $x_{i}$ given $d_{j}(0, \ldots, i)$.

If the nonlinearities of system (9) are sufficiently smooth, this system can be expanded around the state estimate $\hat{x}_{i}$ using Taylor series as:

$\left\{\begin{array}{l}f\left(x_{i}\right)=f\left(\hat{x}_{i}\right)+F_{i} \times\left(x_{i}-\hat{x}_{i}\right)+\text { higher order terms, } \\ h\left(x_{i}\right)=h\left(\hat{x}_{i}\right)+H_{i}^{T} \times\left(x_{i}-\hat{x}_{i}\right)+\text { higher order terms, }\end{array}\right.$

$F_{i}=\left.\frac{\partial f(x)}{\partial x}\right|_{x=\hat{x}_{i}}$,

$H_{i}^{T}=\left.\frac{\partial h(x)}{\partial x}\right|_{x=\hat{x}_{i}}$. 
Neglecting the higher order terms in Eq. (10), Eq. (9) can then be approximated by:

$$
\left\{\begin{aligned}
x_{i} & =F_{i-1} x_{i-1}+\omega_{i-1}+\phi_{i-1} \\
d_{i} & =H_{i}^{T} x_{i}+\nu_{i}+\varphi_{i-1}
\end{aligned}\right.
$$

where

$\phi_{i-1}=f\left(\hat{x}_{i-1}\right)-F_{i-1} \hat{x}_{i-1}$,

$\varphi_{i-1}=h\left(\hat{x}_{i-1}\right)-H_{i-1}^{T} \hat{x}_{i-1}$.

Therefore, the desired estimate $\hat{x}_{i}$ can be obtained by the following EKF recursive equations [26]:

$$
\begin{cases}F_{i-1} & =\left.\frac{\partial f(x)}{\partial x}\right|_{x=\hat{x}_{i-1 \mid i-1}}, \\ H_{i} & =\left.\frac{\partial h(x)}{\partial x}\right|_{x=\hat{x}_{i \mid i-1}}, \\ K_{i} & =P_{i \mid i-1} H_{i}^{T}\left(R_{i}+H_{i} P_{i \mid i-1} H_{i}^{T}\right)^{-1}, \\ \hat{x}_{i \mid i} & =f\left(\hat{x}_{i-1 \mid i-1}\right)+K_{i}\left[d_{i}-h\left(\hat{x}_{i \mid i-1}\right)\right], \\ P_{i \mid i} & =F_{i-1}\left(P_{i-1 \mid i-1}-K_{i} H_{i} P_{i-1 \mid i-1}\right) F_{i-1}^{T}+Q_{i-1},\end{cases}
$$

where $d_{i}$ represents the observation vector, $K_{i}$ is the Kalman gain, $P_{i}$ denotes the covariance matrix of state estimation error, and the estimated state $\hat{x}_{i \mid i}$ is the optimal solution which approaches the conditional mean value $E\left[x_{i} \mid\left(d_{0}, d_{1}, \ldots, d_{i}\right)\right]$.

Ultimately, choosing the state of EKF as $x=\left[U_{f}, \Delta \psi\right]^{T}$, and applying the Kalman recursion (12), the prediction of cross-track error $\hat{\varepsilon}$ can be obtained for the computation of reference heading angle.

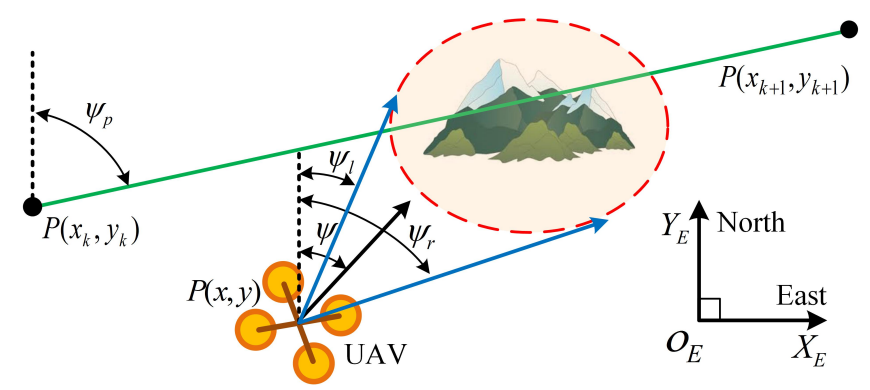

Fig. 4: Geometry of the adopted obstacle avoidance method.

\section{Obstacle Avoidance Scheme Design}

Maintaining the safety of UQHs is generally a priority over mission execution including trajectory tracking and path following. Therefore, in the event of encountering obstacles, the current objective is first to avoid any potential collisions, then return to the assigned mission. Considering the computational capability of the processor onboard UQH and high maneuverability requirement in practice, this study utilizes a relatively simple but effective roadmap-based obstacle avoidance method, the visibility graph, to avoid any obstacles approaching to the UAV.

The general concept of visibility graph method is to connect UAV and the next objective waypoint, two possibility can occur: 1) the next objective waypoint is visible when there is no obstacles between UAV and the waypoint, and the path is approachable; 2) otherwise, the path to objective waypoint is blocked by obstacles, then an optimal path (the

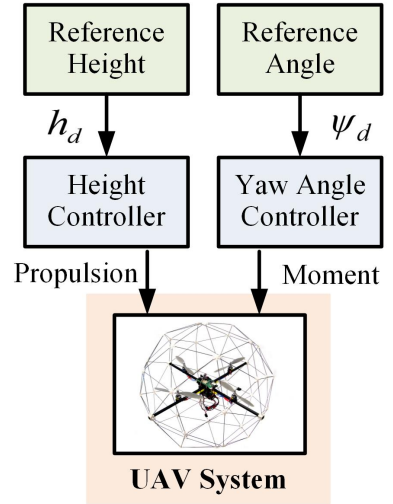

Fig. 5: Composition of controllers to follow the desired path.

shortest path) of avoiding the obstacle and moving towards to objective waypoint is achievable by comparing all of the available connections from UAV, through one of the detected vertex of obstacle, to objective waypoint.

As illustrated in Fig. 4, in the absence of obstacles, UAV follows the desired path by tracking the reference heading angle $\psi_{d}$ generated according to the desired path; but in the presence of obstacles, UAV stops following the desired path and changes to follow the obstacle avoidance heading angle to avoid the approaching obstacles.

In order to calculate the corresponding heading angle to keep the UAV away from colliding with obstacles, the following rule is established:

$$
\psi_{d}= \begin{cases}\psi_{l} & \text { if } \delta_{l} \leq \delta_{r}, \\ \psi_{r} & \text { if } \delta_{l}>\delta_{r},\end{cases}
$$

where $\delta_{l}=\left|\psi_{l}-\psi\right|$ and $\delta_{r}=\left|\psi_{r}-\psi\right| . \psi_{l}$ and $\psi_{r}$ denote the leftmost and rightmost vertex of obstacle combining with a safety distance with respect to UAV, respectively. This safety distance is chosen by inflating the obstacle with a specific radius according to the forward velocity of $\mathrm{UAV}$, minimum turning radius of $\mathrm{UAV}$, and the practical safety requirement.

\section{Control System Design}

As shown in Fig. 5, the tasks of control system design in this study include:

1) To maintain a desired height and follow the reference yaw angle.

2) To keep a constant forward motion by achieving an acceptable performance of pitch angle control.

3) To guarantee the stability of UAV with small deviations from the hovering flight.

A PID controller is chosen for height control, controller gains can be determined by:

$$
u_{z}=K_{P}^{h}\left(z_{d}-z\right)+K_{I}^{h} \int\left(z_{d}-z\right) d t+K_{D}^{h}(-\dot{z}),
$$

where $z_{d}$ is the desired height, and $K_{P}^{h}, K_{I}^{h}$ and $K_{D}^{h}$ are the gains of PID controller. 
A PD controller is designed for the attitude operation (yaw and pitch angle control), controller gains is calculated as:

$$
\begin{aligned}
u_{\theta} & =K_{P}^{\theta}\left(\theta_{d}-\theta\right)+K_{D}^{\theta}\left(p_{d}-p\right), \\
u_{\psi} & =K_{P}^{\psi}\left(\psi_{d}-\psi\right)+K_{D}^{\psi}\left(r_{d}-r\right),
\end{aligned}
$$

where $K_{P}^{\psi}$ and $K_{D}^{\psi}$ are the gains of PD controller for yaw angle, while $K_{P}^{\theta}$ and $K_{D}^{\theta}$ are the gains for pitch angle. $p$ and $r$ denote the angular velocities of pitch and yaw.

In order to improve the robustness of attitude controller against structural changes and uncertainties in system parameters as well as environmental disturbances, the attitude controller is further tuned by the fuzzy logic control (FLC) rule. The following simple linear transformation is used for calculating controller gains:

$$
\begin{aligned}
& K_{P}=\left(K_{P}^{\max }-K_{P}^{\min }\right) \Delta K_{P}+K_{P}^{\min }, \\
& K_{D}=\left(K_{D}^{\max }-K_{D}^{\min }\right) \Delta K_{D}+K_{D}^{\min },
\end{aligned}
$$

where $\left[K_{P}^{\min }, K_{P}^{\max }\right],\left[K_{D}^{\min }, K_{D}^{\max }\right]$ represent the predefined ranges of $K_{P}$ and $K_{D}$, respectively. $\Delta K_{P}$ and $\Delta K_{D}$ are determined by the following linguistic rules of FLC:

If $e_{k}$ is $A_{i}$ and $\Delta e_{k}$ is $B_{i}$, then $\Delta K_{P}$ is $C_{i}$ and $\Delta K_{D}$ is $D_{i}$,

where $A_{i}, B_{i}, C_{i}$, and $D_{i}$ represent the fuzzy sets corresponding to $e_{k}, \Delta e_{k}, \Delta K_{P}$, and $\Delta K_{D}$.

Due to the space limit, further details of the design procedure is omitted here. Readers can refer to other literature for the similar design method [27].

\section{Simulations}

To demonstrate the effectiveness of the proposed method, numerical simulations on a nonlinear UQH model are conducted. Two scenarios are selected in the simulation:

1) Scenario 1: In the first scenario, as shown in Fig. 6, the UAV starts at the left bottom corner $(5 \mathrm{~m}, 20 \mathrm{~m})$, while the end point is located at the right top corner $(250 \mathrm{~m}, 150 \mathrm{~m})$. One static obstacle (the red rectangle) situates at point $(160 \mathrm{~m}, 80 \mathrm{~m})$ of the map..

2) Scenario 2: In this scenario, there are three static obstacles (see Fig. 8) situating at points $(50 \mathrm{~m}, 40 \mathrm{~m})$, $(95 \mathrm{~m}, 60 \mathrm{~m})$, and $(170 \mathrm{~m}, 70 \mathrm{~m})$, respectively.

$\mathrm{UQH}$ is set at a constant forward speed $(10 \mathrm{~m} / \mathrm{s})$ and a constant height. UQH is assumed to be deployed in 2dimensional (2D) space. A straight line starting from $(0,0)$ and ending at $(250 \mathrm{~m}, 150 \mathrm{~m})$ is chosen as the desired path for both scenarios.

\section{A. Results of Scenario 1}

From Fig. 6, it shows that the desired path is followed by the UQH, while the obstacle blocking the path is also successfully avoided, finally, the UQH can return back to the desired path.

Fig. 7 displays the cross-track error is effectively and promptly estimated with an acceptable residual.

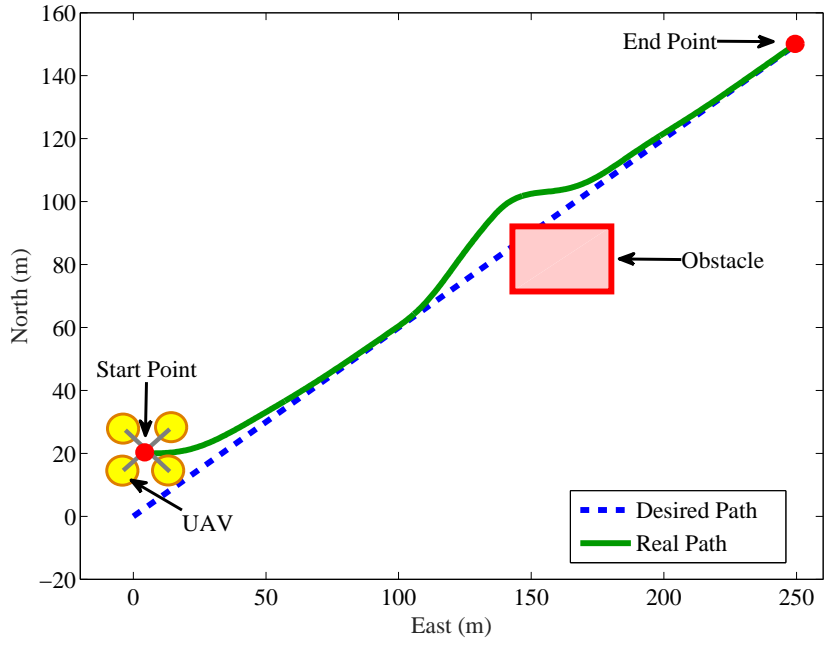

Fig. 6: Performance of path following and obstacle avoidance schemes.

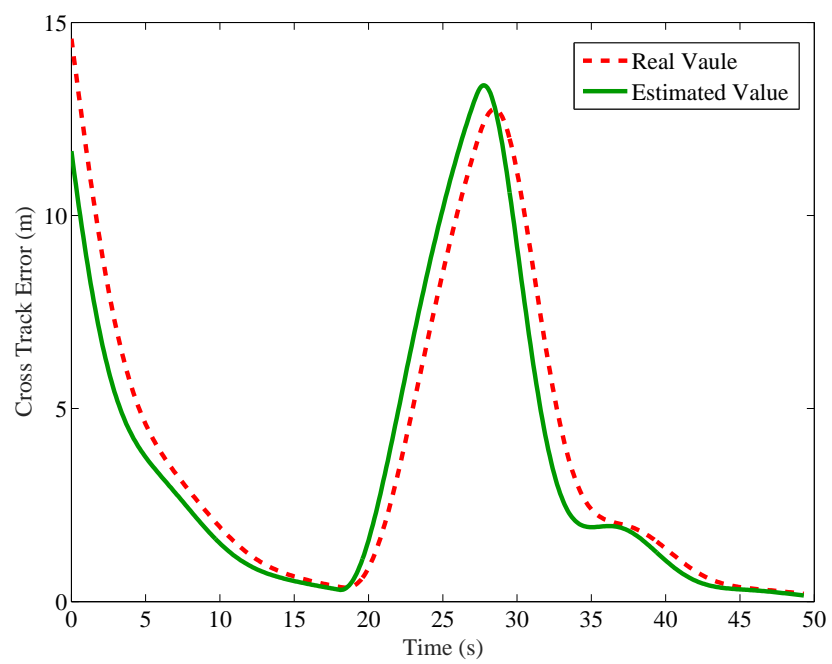

Fig. 7: Performance of cross-track error prediction.

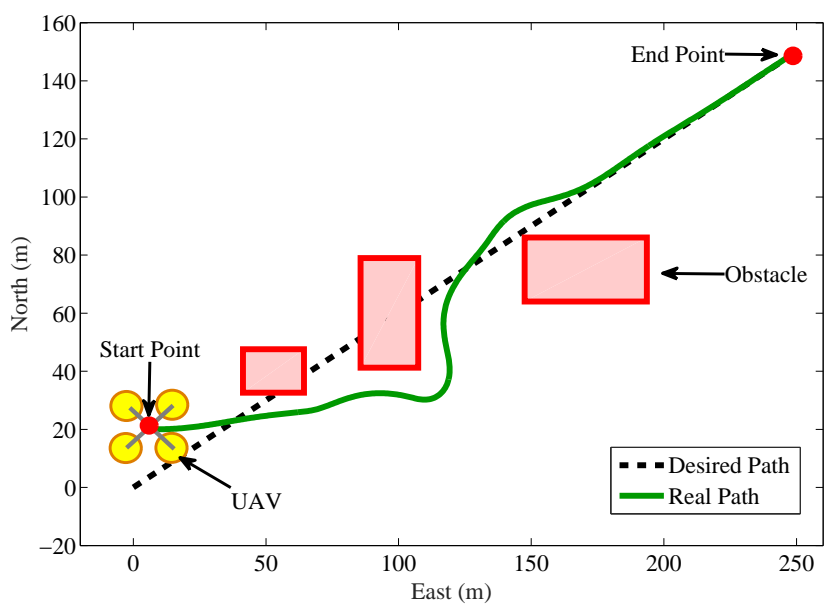

Fig. 8: Performance of path following and obstacle avoidance schemes. 


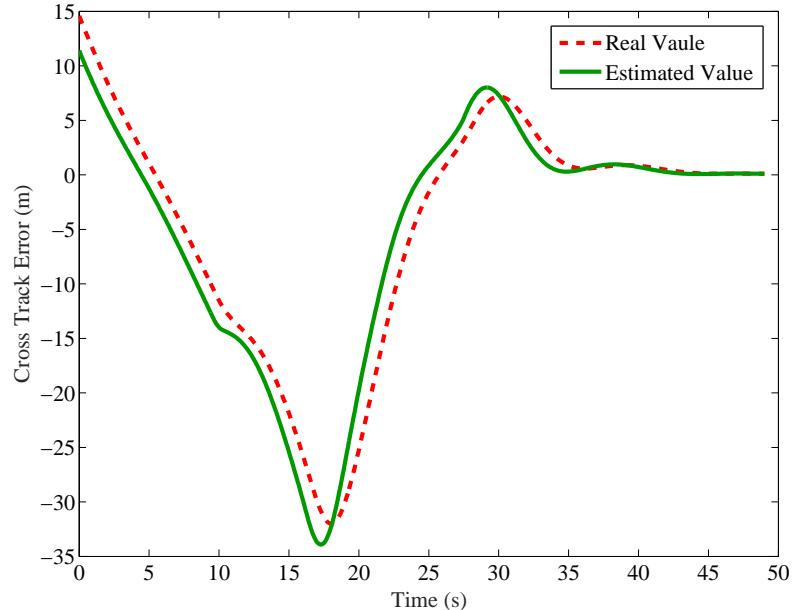

Fig. 9: Performance of cross-track error prediction.

\section{B. Results of Scenario 2}

Fig. 8 shows UQH safely passes the three obstacles, and then continues to path following execution.

As observed from Fig. 9, the cross-track error can still be well estimated even $\mathrm{UQH}$ has experienced a series of sophisticated manipulation.

\section{CONCLUSIONS}

In this paper, a new cross-track error prediction based path following method is presented. Before distributing to the control system, the reference heading angle is generated by the path following module, and to be modified by the obstacle avoidance module in the presence of obstacles. A fuzzy proportional-derivative control approach is then employed for tracking the desired heading angle. Simulations have validated the effectiveness of the proposed approaches.

Potential future work can be extended to the validation of the developed algorithm on a real unmanned quadrotor helicopter in the field test environment. Another possible research direction is suggested to improve the current reference heading angle calculation rule making the path following strategy capable of adapting to various of working and environmental conditions with superior performance.

\section{REFERENCES}

[1] J. E. Gomez-Balderas, G. Flores, L. G. Carrillo, and R. Lozano, "Tracking a ground moving target with a quadrotor using switching control," Journal of Intelligent \& Robotic Systems, vol. 70, no. 1-4, pp. 65-78, 2013.

[2] C. Yuan, Y. M. Zhang, and Z. X. Liu, "A survey on technologies for automatic forest fire monitoring, detection and fighting using UAVs and remote sensing techniques," Canadian Journal of Forest Research, vol. 45, no. 7, pp. 783-792, 2015.

[3] C. Yuan, Z. X. Liu, and Y. M. Zhang, "Aerial images-based forest fire detection for firefighting using optical remote sensing techniques and unmanned aerial vehicles," Journal of Intelligent \& Robotic Systems, 2017, DOI: 10.1007/s10846-016-0464-7.

[4] A. Rango, A. Laliberte, J. E. Herrick, C. Winters, K. Havstad, C. Steele, and D. Browning, "Unmanned aerial vehicle-based remote sensing for rangeland assessment, monitoring, and management," Journal of Applied Remote Sensing vol. 3, no. 1, pp. 33-42, 2009.

[5] O. Shakernia, Y. Ma, T. J. Koo, and S. Sastry, "Landing an unmanned air vehicle: Vision based motion estimation and nonlinear control," Asian Journal of Control, vol. 1, no. 3, pp. 128-145, 1999.
[6] J. Escareño, S. Salazar, H. Romero, and R. Lozano, "Trajectory control of a quadrotor subject to 2D wind disturbances," Journal of Intelligent \& Robotic Systems, vol. 70, no. 1-4, pp. 51-63, 2013.

[7] Z. X. Liu, C. Yuan, Y. M. Zhang, and J. Luo, "A learning-based fault tolerant tracking control of an unmanned quadrotor helicopter," Journal of Intelligent \& Robotic Systems, pp. 1-18, 2015.

[8] K. Alexis, G. Nikolakopoulos, and A. Tzes, "Switching model predictive attitude control for a quadrotor helicopter subject to atmospheric disturbances," Control Engineering Practice, vol. 19, no. 10, pp. 11951207, 2011.

[9] Y. M. Zhang, A, Chamseddine, C. A. Rabbath, B. W. Gordon, C. Y. Su, S. Rakheja, C. Fulford, J. Apkarian, and P. Gosselin, "Development of advanced FDD and FTC techniques with application to an unmanned quadrotor helicopter testbed," Journal of Franklin Institute, vol. 350, no. 9, pp. 2396-2422, 2013.

[10] P. Castillo, A. Dzul, and R. Lozano, "Real-time stabilization and tracking of a four-rotor mini rotorcraft," IEEE Transactions on Control Systems Technology, vol. 12, no. 4, pp. 510-516, 2004.

[11] T. Dierks, and S. Jagannathan, "Output feedback control of a quadrotor UAV using neural networks," IEEE Transactions on Neural Networks, vol. 21, no. 1, pp. 50-66, 2010.

[12] G. M. Hoffmann, H. Huang, S. L. Waslander, and C. J. Tomlin, "Precision flight control for a multi-vehicle quadrotor helicopter testbed," Control Engineering Practice, vol. 19, no. 9, pp. 1023-1036, 2011.

[13] Z. X. Liu, Y. M. Zhang, X. Yu, and C. Yuan, "Unmanned surface vehicles: An overview of developments and challenges," Annual Reviews in Control, vol. 41, pp. 71-93, 2016.

[14] L. Consolini, M. Manfredi, N. Christopher, and T. Mario, "Path following for the PVTOL aircraft," Automatica, vol. 46, no. 8, pp. 1284-1296, 2010.

[15] P. Sanghyuk, J. Deyst, and J. P. How, "Performance and Lyapunov stability of a nonlinear path following guidance method," Journal of Guidance, Control, and Dynamics, vol. 30, no. 6, pp. 1718-1728, 2007.

[16] D. K. Duc, Z. P. Jiang, and J. Pan, "On global tracking control of a VTOL aircraft without velocity measurements," IEEE Transactions on Automatic Control, vol. 48, no. 12, pp. 2212-2217, 2003.

[17] D. R. Nelson, D. B. Barber, T. W. McLain, and R. W. Beard, "Vector field path following for miniature air vehicles," IEEE Transactions on Robotics, vol. 23, no. 3, pp. 519-529, 2007.

[18] I. Kaminer, A. Pascoal, E. Xargay, N. Hovakimyan, C. Cao, and V. Dobrokhodov, "Path following for small unmanned aerial vehicles using L1 adaptive augmentation of commercial autopilots," Journal of Guidance, Control, and Dynamics, vol. 33, no. 2, pp. 550-564, 2010.

[19] J. L. Foo, J. Knutzon, V. Kalivarapu, J. Oliver, and E. Winer, "Path planning of unmanned aerial vehicles using B-splines and particle swarm optimization," Journal of Aerospace Computing, Information, and Communication, vol. 6, no. 4, pp. 271-290, 2009.

[20] L. C. Lai, C. C. Yang, and C. J. Wu, "Time-optimal control of a hovering quad-rotor helicopter," Journal of Intelligent \& Robotic Systems, vol. 45, no. 2, pp. 115-135, 2006.

[21] A. Franchi, C. Secchi, M. Ryll, H. H. Bulthoff, and P. R. Giordano, "Shared control: Balancing autonomy and human assistance with a group of quadrotor UAVs," IEEE Robotics \& Automation Magazine, vol. 19 , no. 3 pp. 57-68, 2012

[22] Z. X. Liu, C. Yuan, X. Yu, and Y. M. Zhang, "Fault-tolerant formation control of unmanned aerial vehicles in the presence of actuator faults and obstacles," Unmanned Systems, vol. 4, no. 3, pp. 197-211 2016.

[23] S. Hrabar, "3D path planning and stereo-based obstacle avoidance for rotorcraft UAVs," In IEEE/RSJ International Conference on Intelligent Robots and Systems, pp. 807-814, 2008.

[24] L. P., Tomás, and M. A. Wesley, "An algorithm for planning collisionfree paths among polyhedral obstacles," Communications of the ACM 22, vol. 22, no. 10, pp. 560-570, 1979.

[25] K. K. Ahn, and D. Q. Truong, "Online tuning fuzzy PID controller using robust extended Kalman filter," Journal of Process Control, vol. 19, no. 6, pp. 1011-1023, 2009.

[26] M. S. Grewal, A. P. Andrews, "Kalman filtering: Theory and practice using MATLAB," John Wiley \& Sons, 2011.

[27] M. H. Amoozgar, A. Chamseddine, and Y. M. Zhang, "Fault-tolerant fuzzy gain-scheduled PID for a quadrotor helicopter testbed in the presence of actuator faults," IFAC Proceedings Volumes, vol. 45, no. 3, pp. 282-287, 2012. 